\title{
ÍNDICES GENÉTICOS ESTIMADOS PARA PESO CORPORAL EN LLAMAS
}

\author{
ESTIMATED GENETIC INDEXES FOR BODY WEIGHT IN LAMAS \\ Wilber García V. ${ }^{1}$ y Víctor Leyva V. ${ }^{2}$
}

\section{Resumen}

\begin{abstract}
Se utilizó información de parición, destete y esquila de llamas Cha'ccus y K’aras, de los registros de la Estación Experimental del Centro de Investigaciones IVITA-Maranganí del periodo 1995-2001. Se estimó la heredabilidad $\left(\mathrm{h}^{2}\right)$, repetibilidad y correlaciones genéticas de peso corporal al nacimiento, destete, y primera y segunda esquila ajustando los datos por efecto del año de producción, edad de la madre y sexo de la cría. Los valores de $\mathrm{h}^{2}$ se estimaron a través de un diseño de hermanos enteros, de medios hermanos paternos y regresión cría madre. $\mathrm{La} \mathrm{h}^{2}$ para el peso al destete, tanto en llamas $\mathrm{K}^{\prime}$ ara como en Cha'ccus fue alta, mientras que para el peso al nacimiento y peso vivo a la primera y segunda esquila tuvo valores de medio a alto. Los índices de repetibilidad para el peso vivo en llamas Cha'ccus y $\mathrm{K}^{\prime}$ aras, estimados a través del método de la correlación intraclase fueron inferiores (0.3-0.4) que aquellos estimados a través del método de la regresión (0.6-0.7, respectivamente). Las correlaciones genéticas entre peso vivo al nacimiento con peso vivo al destete y primera esquila, y entre peso vivo al destete y peso vivo a la primera esquila presentaron valores altos y positivos en ambas variedades. Estos estimados son confiables para elegir el método de selección por índices en un plan de mejoramiento genético para la producción de carne.
\end{abstract}

Palabras clave : llamas, índices genéticos, peso vivo

\section{ABSTRACT}

Parturition, weaning and shearing data of K'aras and Ch'accus lamas, collected from 1995 to 2001 at the IVITA Research Center, Maranganí of San Marcos University, was used in the present study to estimate the heritability $\left(\mathrm{h}^{2}\right)$, repeatability and genetic correlations between body weight at birth, weaning and first and second shearing, by adjusting the fata for the effect of year, age of mother and sex of baby lama. The $\mathrm{h}^{2}$ value was analyzed by the nested effect using a model for brothers, brothers in law and by regression baby-mother. Heritability for body weight for both $\mathrm{K}^{\prime}$ aras and Ch'accus lamas was high, while for birth weight and weight at first and second shearing varied from medium to high. The repeatability estimated by intraclass correlation for body weight of Ch'accus and $\mathrm{K}^{\prime}$ aras was lower (0.3-0.4) than those estimated by regression (0.6-0.7, respectively). Genetic correlations between body weight at birth and at weaning and first shearing, and between body weight at weaning and at first shearing wee high and positive

\footnotetext{
${ }^{1}$ Estación Experimental del Centro de Investigaciones IVITA-Maranganí, FMV-UNMSM

${ }^{2}$ Laboratorio de Reproducción Animal, FMV-UNMSM.E-mail: vleyvav@unmsm.edu.pe
} 
in both strains. These estimations are reliable to choose the appropriate selection index method for a genetic improvement program in lama meat production.

Key words: lama, genetic index, body weight

\section{INTRODUCCIÓN}

La población nacional de llamas en el Perú es estimada en 1'462,730 cabezas (INEI, 2005). De esta población, el 5 al 10\% se encuentra bajo una crianza de tipo empírica y mayormente asociada con alpacas y ovinos. La llama no tiene un objetivo productivo definido, a excepción de su empleo como animal de carga y del uso de su fibra para la elaboración de sacos y soguillas. Estos animales son beneficiados cuando dejan de ser útiles para estas actividades, de allí que su aporte como animal productor de carne es muy limitado (Leyva, 1990).

La llama posee cualidades importantes, como un rendimiento de carcasa entre $58 \mathrm{y}$ 60\% (Bravo et al., 1981; Bustinza, 1998), una eficiente utilización y conversión de los pastos de baja calidad, con un menor requerimiento para mantenimiento comparado con la del ovino (San Martín, 1996) y una capacidad en producción de leche mayor que la alpaca (Leyva et al., 1983b) con niveles sostenidos después del pico de producción (Leyva, 1983a; Novoa y Leyva, 1996), con un efecto positivo en la tasa de crecimiento de su cría.

Estas características apoyan el propósito de orientar la crianza de llamas a la producción de carne, y con este fin la Estación Experimental del Centro de Investigaciones IVITA-Maranganí estableció un núcleo de reproductores siguiendo un proceso de selección en base al comportamiento productivo de los animales y sus progenies. Parte de esta evaluación ha sido publicada (García et al., 1998, 2002). El éxito de estos programas genéticos depende de las mejoras de los parámetros productivos de las crías, y para ello se requiere conocer los índices de heredabilidad, repetibilidad, así como las correlaciones genéticas, siendo el propósito del presente estudio la obtención de estos índices en llamas de las variedades Cha'ccu y $\mathrm{K}^{\prime}$ ara.

\section{Materiales y Métodos}

El estudio se llevó a cabo con los datos del Núcleo de Reproductores Llamas de la Estación Experimental del Centro de Investigaciones IVITA-Maranganí, ubicado en el "Fundo San Luis" del distrito de Nuñoa, provincia de Melgar, Puno, a una altitud de 4300 a $4500 \mathrm{msnm}$. El sistema de crianza fue en pastoreo de tipo extensivo en pradera nativa, en laderas y pampas donde existía predominancia de Festuca dolichophila, Mulhembergia fastigiata y Alchemilla pinnata, así como en zonas de bofedales donde predominaba la Distichia muscoides, Eleocharis albibractiata y Alchemilla diplophylla.

Se utilizó el método de "empadre controlado" para la cubrición de las llamas durante la estación de monta. Se registró el fenotipo del binomio madre-cría y los datos del parto; además del peso al destete y luego de la primera y segunda esquila, la cual se realiza cada dos años.

Se analizó los registros del periodo 19952001, que incluyó 1200 nacimientos, 800 destetes (edad promedio: 7 meses), y 700 y 600 observaciones de primera y segunda esquila. Se hizo un ajuste por fecha de nacimiento para el análisis de las variables peso vivo al destete y a la primera y segunda esquila, utilizando las fórmulas de Warwick y Legates (1980). 
Se utilizó la clasificación anidada "modelo madre" (Yij $=\mu+\alpha i+\beta j+$ eij) y "modelo progenie" (Yijk $=\mu+\alpha i+\beta j+\delta k+$ eijk $)$ bajo un diseño completamente al azar, para corregir la influencia de los efectos considerados como no genéticos de variación, tales como el año de producción, sexo de la cría y edad de la madre. Se utilizó la correlación intraclase para estimar la repetibilidad, con el modelo Yij $=\mu+\alpha i+\delta j+$ eij, donde $\alpha i=$ efecto animal, $\delta \mathrm{j}=$ efecto año, y eij $=$ desviación ambiental. Los datos estandarizados fueron ajustados por un factor de corrección estimado por el método de mínimos cuadrados (Larson y Fernández 1983) usando el paquete estadístico Statistical Análisis System (SAS). La estimación de los índices genéticos se hizo con los datos ajustados.

\section{Resultados y Discusión}

\section{Ajuste por el defecto de causas no genéticas de variación}

El año de nacimiento de las crías afectó significativamente el peso vivo al nacimiento, al destete y a la primera y segunda esquila, lo que implica que el medio ambiente tiene una influencia marcada sobre el desarrollo de la biomasa corporal pre y postparto de las llamas a través del tiempo. Quirita (1991) y Olarte et al. (1999) indican haber encontrado diferencias significativas en el peso vivo a la primera esquila en alpacas por efecto del año de nacimiento.

El factor sexo de la cría no influyó en el peso al nacimiento y al destete, pero sí afectó el peso a la primera y segunda esquila, observándose que los machos son más pesados; sin embargo, Condorena (1980) encontró que las alpacas hembras tienen mayor o igual peso que los machos al destete, al año ( $1^{\text {ra }}$ esquila) y dos años de edad (2 $2^{\text {da }}$ esquila). Esta diferencia se atribuye en la alpaca hembra al efecto hormonal que estimula el inicio temprano de su actividad sexual (1 año) a través de un mayor desarrollo corporal (Novoa et al., 1972), ya que los machos inician su vida reproductiva a los 3 años (Novoa, 1981). El factor edad de la madre influyó sobre el peso vivo al nacimiento y al destete, siendo las llamas de mayor edad (7 a 13 años) las que tiene crías más pesadas. Las llamas de mayor edad y de varios partos tienen mayor espacio uterino para un mayor desarrollo del feto y mayor peso al nacimiento de la cría (Puma et al., 1999), además que tienen una mayor producción de leche, lo cual resulta en una mayor tasa de crecimiento de sus crías (Leyva et al., 1983a,b). Sin embargo, este efecto no influyó sobre el peso vivo en la primera y segunda esquila, aunque hay reportes en alpacas que indican lo contrario (Olarte, 1999).

\section{Heredabilidad para características de peso vivo}

El Cuadro 1 muestra los valores de índices de herencia para las características asociadas al peso vivo al nacimiento, al destete y a la primera y segunda esquila. Estos valores para peso al nacimiento de llamas Cha'ccu y K'ara son diferentes a otros reportes (Cuadro 2). Los índices de herencia para el peso vivo al destete, determinados a través de los tres métodos, fueron considerados como de heredabilidad muy alta en llamas K'ara y de heredabilidad alta en la variedad Cha'ccu.

Los índices de herencia para la característica peso corporal a la primera esquila en llamas, estimados por los tres métodos, se consideran como de heredabilidad alta. Choque y Rodríguez (1998) reportan valores inferiores (Cuadro 2), posiblemente debido al número de animales, espacio geográfico, sistema de crianza y diferencia de variedades. Por otro lado, no se encuentra en la literatura valores referenciales para el índice de herencia para peso vivo a la segunda esquila. 
Cuadro 1. Índices de herencia $\left(\mathrm{h}^{2} \pm\right.$ e.e.) para el peso corporal en diferentes etapas de la vida productiva de llamas Cha'ccu y K'ara criadas en el Altiplano peruano

\begin{tabular}{lccc}
\hline Peso vivo & $\begin{array}{c}\text { Observaciones } \\
(\mathrm{n})\end{array}$ & Cha'ccu & K'ara $^{\prime}$ \\
\hline Al nacimiento & & & \\
Hermanos enteros & 1200 & $0.354 \pm 0.458$ & $0.489 \pm 0.451$ \\
Medios hermanos & 1200 & $0.451 \pm 0.188$ & $0.510 \pm 0.245$ \\
Regresión cría madre & & $0.588 \pm 0.081$ & $0.598 \pm 0.021$ \\
Al destete & & & \\
Hermanos enteros & 800 & $0.588 \pm 0.095$ & $0.645 \pm 0.545$ \\
Medios hermanos & 800 & $0.410 \pm 0.141$ & $0.610 \pm 0.225$ \\
Regresión cría madre & & $0.580 \pm 0.205$ & $0.680 \pm 0.124$ \\
A la 1 ${ }^{\text {e esquila }}$ & & & \\
Hermanos enteros & 700 & $0.435 \pm 0.125$ & $0.405 \pm 0.158$ \\
Medios hermanos & 700 & $0.499 \pm 0.191$ & $0.501 \pm 0.215$ \\
Regresión cría madre & & $0.510 \pm 0.258$ & $0.591 \pm 0.131$ \\
A la 2 ${ }^{\text {a }}$ esquila & & & \\
Hermanos enteros & 600 & $0.280 \pm 0.131$ & $0.305 \pm 0.322$ \\
Medios hermanos & 600 & $0.325 \pm 0.241$ & $0.360 \pm 0.720$ \\
Regresión cría madre & & $0.358 \pm 0.231$ & $0.370 \pm 0.138$ \\
\hline
\end{tabular}

Cuadro 2. Índices de herencia $\left(\mathrm{h}^{2} \pm\right.$ e.e.) reportados en la literatura para el peso corporal en diferentes etapas de la vida productiva de llamas y alpacas criadas en el Altiplano peruano

\begin{tabular}{|c|c|c|c|c|}
\hline Peso corporal & Especie & Método de análisis & $h^{2} \pm$ e.e. & Referencia \\
\hline \multirow{4}{*}{ Al nacimiento } & \multirow{3}{*}{ Llama } & Hermanos enteros & $0.047 \pm 0.24$ & Choque y Rodríguez (1998) \\
\hline & & Medios hermanos & $\begin{array}{l}0.45 \pm 0.24 \\
0.34 \pm 0.23\end{array}$ & $\begin{array}{l}\text { Choque y Rodríguez (1998) } \\
\text { Bustinza (1991) }\end{array}$ \\
\hline & & Regresión cría-madre & $0.14 \pm 0.12$ & Choque y Rodríguez (1998) \\
\hline & Alpaca & Regresión cría-madre & $\begin{array}{l}0.53 \pm 0.11 \\
0.32 \pm 0.20\end{array}$ & $\begin{array}{l}\text { Bravo y Velasco (1983) } \\
\text { Quirita (1991) }\end{array}$ \\
\hline \multirow{2}{*}{ Al destete } & Llama & $\begin{array}{l}\text { Hermanos enteros } \\
\text { Regresión cría-madre }\end{array}$ & $\begin{array}{l}0.047 \pm 0.24 \\
0.14 \pm 0.12\end{array}$ & Choque y Rodríguez (1998) \\
\hline & Alpaca & Regresión cría-madre & $\begin{array}{l}0.41 \pm 0.14 \\
0.34\end{array}$ & $\begin{array}{l}\text { Quirita (1991) } \\
\text { Bravo y Velasco (1983) }\end{array}$ \\
\hline A la $1^{\mathrm{a}}$ esquila & Llama & $\begin{array}{l}\text { Hermanos enteros } \\
\text { Medios hermanos } \\
\text { Regresión cría-madre }\end{array}$ & $\begin{array}{l}0.36 \pm 0.33 \\
0.30 \pm 0.59 \\
0.53 \pm 0.03\end{array}$ & Choque y Rodríguez (1998) \\
\hline
\end{tabular}


Cuadro 3. Repetibilidad ( $\mathrm{R} \pm$ e.e.) para el peso corporal de llamas Cha'ccu y K'ara criadas en el Altiplano peruano, estimada por el método de la regresión y la correlación intraclase

\begin{tabular}{lccc}
\hline Carácter & $\begin{array}{c}\text { Observaciones } \\
(\mathrm{n})\end{array}$ & Cha'ccu & K'ara \\
\hline Regresión & 300 & $0.638 \pm 0.065$ & $0.710 \pm 0.101$ \\
Correlación & 700 & $0.321 \pm 0.110$ & $0.356 \pm 0.115$ \\
\hline
\end{tabular}

Cuadro 4. Correlaciones genéticas $(\mathrm{R} \pm$ e.e.) para características de peso vivo en llamas Cha'ccu y K'ara criadas en el Altiplano peruano

\begin{tabular}{rcc}
\hline Peso & Cha'ccu & K'ara \\
\hline Al nacimiento & & \\
Destete & $0.631 \pm 0.295$ & $0.550 \pm 0.530$ \\
$1^{\text {a }}$ esquila & $0.510 \pm 0.284$ & $0.540 \pm 0.740$ \\
$2^{\text {a }}$ esquila & $0.380 \pm 0.580$ & $0.430 \pm 0.428$ \\
Al destete & & \\
$1^{\text {a }}$ esquila & $0.560 \pm 0.180$ & $0.490 \pm 0.290$ \\
$2^{\text {a }}$ esquila & $0.380 \pm 0.187$ & $0.045 \pm 0.090$ \\
A la $1^{\text {a }}$ esquila & & $0.142 \pm 0.228$ \\
$2^{\text {a }}$ esquila & $0.163 \pm 0.430$ & \\
\hline
\end{tabular}

\section{Índice de Repetibilidad (R)}

El índice de repetibilidad para peso vivo de la población de llamas, estimado mediante el método de regresión de los valores del comportamiento futuro sobre el comportamiento pasado y la estimación de la correlación intraclase, se presenta en el Cuadro 3.

Los valores de índice de repetibilidad para peso vivo, estimados a través de la regresión, respecto de aquellos estimados por correlación, son superiores debido a que el efecto año afecta significativamente a esta característica y esto no puede ser controlado por el modelo de regresión. Estos valores son superiores a los reportados por Rodríguez e Iñiguez (1974), quienes indicaron un valor de $\mathrm{R}=0.224$, dada la gran variabilidad fenotípica de sus animales, ya que no consideraron la diferencia entre las dos variedades. Otros autores, utilizando el método de regresión en alpacas, obtuvieron valores similares al presente estudio (Mamani, 1991).

\section{Correlaciones genéticas}

Las correlaciones genéticas entre las características de peso vivo al nacimiento con peso vivo al destete y peso vivo a la primera 
esquila son altos y positivos (Cuadro 4), lo cual podría atribuirse a la producción de leche de la madre, ya que se ha demostrado una correlación significativa con la tasa de crecimiento de la cría (Gonzáles et al., 2007). En ovinos se ha demostrado que el peso corporal y el rendimiento de carcasa al destete dependió de la relación peso corporal al nacimiento y producción de leche (Geenty, 1979; Torres-Hernandez y Hohenboken, 1980).

Los resultados obtenidos en el presente estudio son iguales a los reportados en alpacas por Quirita et al. (1991), aunque inferiores a valores reportados en ovinos Merino y Hampshire entre peso vivo al destete con peso vivo a la primera esquila $(0.97$ y 0.72 , respectivamente) (Young et al., 1965; Dzakuma et al., 1978). En base a estos resultados y a reportes previos, se puede inferir que la selección por el carácter peso vivo al nacimiento influiría en la selección indirecta del carácter peso vivo al destete y a la primera esquila.

\section{Conclusiones}

- Los índices de heredabilidad para peso al destete en llamas Cha'ccu y K'ara determinados a través de los métodos correlación intraclase de hermanos enteros, medios hermanos y regresión cría madre tienen valores altos.

- Los índices de repetibilidad para la característica peso vivo en llamas, estimados a través de la correlación intraclase, dan valores medios, en comparación de aquellos obtenidos por el método de la regresión que son altos.

- Las correlaciones genéticas entre peso al nacimiento con peso al destete y a la primera esquila, y entre peso al destete con peso a la primera esquila son altos en ambas variedades.

\section{Literatura Citada}

1. Bravo, W.; E. Franco; J. Sumar. 1981. Rendimiento de canal en llamas.
Resúmenes $4^{\text {ta }}$ Convención Internacional sobre Camélidos Sudamericanos. Punta Arenas, Chile. p 60.

2. Bravo, W; J. Velasco. 1983. Índice de herencia del peso al nacimiento, destete y la primera esquila en alpacas. Resúmenes VII Congreso Nacional de Ciencias Veterinarias. Ica, Perú.

3. Bustinza, V. 1991. Mejoramiento genético. En: Producción de rumiantes menores: alpaca. Novoa, C.; A. Flores (eds). RERUMEN. Lima, Perú. p 113-128.

4. Bustinza, V. 1998. La llama. Fenotipos y producción. Instituto de Investigación y Promoción de Camélidos Sudamericanos. Ed. FMVZ-UNA-Puno. 51 p.

5. Choque, F.; T. Rodríguez. 1998. Determinación de parámetros genéticos en caracteres de producción de carne y fibra en llamas. VI Congreso Internacional de Especialistas en Camélidos Sudamericanos. Oruro, Bolivia. p 35.

6. Condorena, N. 1980. Algunos índices de producción de alpacas bajo sistema de esquila anual establecido en La Raya. Rev. Inv. Pec. (IVITA) 5(1): 50-54.

7. Dzakuma, J.; N. Nielsen; T. Doane. 1978. Genetic and phenotipic parameter estimates for growth and wool traits in Hampshire sheep. J. Anim. Sci. 47: 10141021.

8. García, W.; D. Pezo; E. Franco; F. San Martín; C. Novoa. 1998. Estudio de la productividad de un núcleo de reproductores llamas de la puna húmeda del Perú. XXI Reunión APPA. Puno, Perú. p 357-360.

9. García, W.; F. San Martín; C. Novoa; E. Franco. 2002. Engorde de llamas bajo diferentes regímenes alimenticios. Rev. Inv. Vet. Perú 13(2): 1-9.

10. Geenty, K.G. 1979. Effect of weaning age on export lamb production. Proc. New Zealand Soc. Anim. Prod. 39: 202-210.

11. Gonzales, R.; V. Leyva; W. García; C. Gavidia; D. Ticona. 2007. Efecto de la alimentación sobre la producción láctea en llamas seleccionadas para producción de carne. Rev. Inv. Vet. Perú 18: 30-39. 
12. [INEI] Instituto Nacional de Estadística e Informática. 2005. Compendio Estadistico. Lima, Perú. p 462.

13. Larson, J. S. Fernández. 1983. Introducción a la teoría de probabilidades e inferencias estadísticas. Ed. Limusa. México. $466 \mathrm{p}$.

14. Leyva, V. 1990. Informe de la VIII Reunión de Trabajo sobre Sistemas de Producción Animal. Centro de Investigación Internacional para el Desarrollo (CIID). IDRC-MR139s: 143-169.

15. Leyva, V.; E. Franco; N. Condorena. 1983a. Determinación de la curva lactacional de alpacas y llamas bajo condiciones de pastura natural. VI Reunión APPA. Lambayeque. Perú.

16. Leyva, V.; E. Franco; N. Condorena. 1983b. Patrón lactacional de alpacas y llamas bajo condiciones de pastura natural. Proyecto de Desarrollo de la Crianza de Alpaca. Convenio IVITACOTESU. Perú. p 79-82.

17. Mamani, G. 1991. Parámetros genéticos del peso vivo y vellón en alpacas. VII Convención Internac. de Especialistas en Camélidos Sudamericanos. Jujuy, Argentina.

18. Novoa, C. 1981. Reproducción de alpacas. En: Producción de rumiantes menores: alpacas. Ed. Novoa C.; A. Florez. RERUMEN. p 103-112.

19. Novoa, C.; S. Fernández-Baca; J. Sumar; V. Leyva. 1972. Pubertad en la alpaca. Rev. Inv. Pec. IVITA 1(1): 29-35.

20. Novoa, C.; V. Leyva. 1996. Reproducción en alpacas y llamas. Publ. Cient. IVITA No. $26.30 \mathrm{p}$.
21. Olarte, U.; W. Zea; B. Ibañez. 1999. Índices de selección y ganancia genética esperada en el mejoramiento de alpaca. II Congreso Mundial sobre Camélidos. p 66.

22. Puma, G.; J. Garnica; P.W. Bravo. 1999. El tamaño de la placenta, edad de la madre y la sobrevivencia perinatal de la cría alpaca. II Congreso Mundial Sobre Camélidos. Cusco, Perú. p 103.

23. Quirita, C. 1991. Estimación de parámetros genéticos en alpacas Huacaya del Centro Experimental La Raya de la UNSAC. Tesis de Ingeniero Zootecnista. Facultad de Ingeniería Zootecnia, Univ. Nacional San Antonio Abad. Cusco, Perú. 48 p.

24. Rodríguez, T.; L. Iñiguez. 1974. Estimación de la vida productiva y la influencia de algunos factores ambientales específicos en llamas. III Reunión de Ganadería. Tarija, Bolivia.

25. San Martín, F. 1996. Nutrición en alpacas y llamas. Pub. Cient. IVITA No. 27. Facultad de Medicina Veterinaria, UNMSM. Lima. 29p.

26. Torres-Hernández, G.; Hohenboken. 1980. Relationship between ewe milk production and composition and preweaning lamb weight gain. J. Anim. Sci. 50: 597-603.

27. Young, S.; G. Brown; H. Turner; C. Dolling. 1965. Genetic and Phonotypic parameters for body weight and greasy fleece weight at weaning in Australian Merino sheep. Aust. J. Agric. Res. 16: 997.

28. Warwick. J.; J. Legates. 1980. Cría y mejora del ganado. $3^{\mathrm{a}}$ ed. Interamericana McGraw-Hill. México. 623 p. 